\title{
INTERPOLATION SEQUENCES FOR THE BERNSTEIN ALGEBRA
}

\author{
XAVIER MASSANEDA AND JOAQUIM ORTEGA-CERDÀ
}

\begin{abstract}
We give a description, in analytic and geometric terms, of the interpolation sequences for the algebra of entire functions of exponential type which are bounded on the real line.
\end{abstract}

\section{Dedicated to Victor Petrovich Havin in his 75 birthday}

\section{INTRODUCTION}

In this paper we describe interpolation sequences for the Bernstein algebra $\mathcal{B}$ of entire functions of exponential type which are bounded on the real line.

The space $\mathcal{B}$ consists of the entire functions $f$ such that for some $A, B>$ 0

$$
\log |f(z)| \leq A+B|\operatorname{Im} z| \quad z \in \mathbb{C} .
$$

It can also be viewed as the union of the Bernstein spaces $B_{\sigma}, \sigma>0$, of entire functions $f$ such that

$$
\sup _{z \in \mathbb{C}}|f(z)| e^{-\sigma|\operatorname{Im} z|}<\infty,
$$

which are precisely the bounded functions with Fourier-Laplace transforms supported in $[-\sigma, \sigma]$.

We were motivated to study the interpolation in $\mathcal{B}$ by the recent characterisation of zero sets in the same class obtained by Sergei Ju. Favorov.

Given a discrete sequence $\Lambda \subset \mathbb{C}$ and a point $z \in \mathbb{C}$, consider the counting function $n_{\Lambda}(z, t)=\#(\Lambda \cap \overline{D(z, t)}), t>0$.

Theorem. [Fa07, Theorem 2.2] A sequence $\Lambda$ is the zero set of a function in $\mathcal{B}$ if and only if:

(a) $\exists \lim _{R \rightarrow \infty} \sum_{\lambda \in \Lambda \cap D(0, R) \backslash\{0\}} \lambda^{-1}$

(b) $n_{\Lambda}(0, t)=O(t), t \rightarrow \infty$

Date: August 8, 2008.

1991 Mathematics Subject Classification. 30E05, 42A85.

Key words and phrases. interpolation sequence, Bernstein spaces.

Both authors supported by DGICYT grant MTM2005-008984-C02-02 and the CIRIT grant 2005-SGR 00611. 
(c) $n_{\Lambda}(0, t+1)-n_{\Lambda}(0, t)=o(t), t \rightarrow \infty$

(d) $\sup _{x \in \mathbb{R}} \int_{0}^{\infty}\left[n_{\Lambda}(b, t)-n_{\Lambda}(x, t)\right] \frac{d t}{t}<\infty$ for some $b \in \mathbb{R} \backslash \Lambda$.

Notice that a subset of a zero set $\Lambda$ of $\mathcal{B}$ is not necessarily a zero set of $\mathcal{B}$, since conditions (a) and (d) imply a certain regularity in the distribution of $\Lambda$ that can be destroyed when removing points.

In view of this resulted we ask ourselves whether interpolation sequences for $\mathcal{B}$ can be described in similar terms. Given the characteristic growth of functions in $\mathcal{B}$, the natural interpolation problem for this algebra is the following.

Definition. A discrete sequence $\Lambda$ in $\mathbb{C}$ is called an interpolation sequence for $\mathcal{B}$ if for every sequence of values $\left\{v_{\lambda}\right\}_{\lambda \in \Lambda}$ with

$$
\sup _{\lambda \in \Lambda}\left|v_{\lambda}\right| e^{-C|\operatorname{Im} \lambda|}<\infty
$$

for some $C>0$, there exists $f \in \mathcal{B}$ with

$$
f(\lambda)=v_{\lambda} \quad \lambda \in \Lambda .
$$

Letting $\mathcal{B}(\Lambda)$ denote the space of sequences $\left\{v_{\lambda}\right\}_{\lambda \in \Lambda}$ satisfying (1) for some $C>0$, we can equivalently define interpolating sequences as those such that the restriction operator

$$
\begin{aligned}
\mathcal{R}_{\Lambda}: \mathcal{B} & \longrightarrow \mathcal{B}(\Lambda) \\
f & \mapsto\{f(\lambda)\}_{\lambda \in \Lambda}
\end{aligned}
$$

is onto.

Applying the open mapping theorem to $\mathcal{R}_{\Lambda}$ [BrGa95, Lemma 2.2.6] one sees that the interpolation is stable in the following sense: for each $C>0$ there exist $M, \sigma>0$ such that if $\left\{v_{\lambda}\right\}_{\lambda \in \Lambda}$ satisfies (1) then there exists $f \in \mathcal{B}$ with $f(\lambda)=v_{\lambda}, \lambda \in \Lambda$, and $\sup _{z \in \mathbb{C}}|f(z)| e^{-\sigma|\operatorname{Im} z|} \leq$ $M \sup _{\lambda \in \Lambda}\left|v_{\lambda}\right| e^{-C|\operatorname{Im} \lambda|}$.

There is an alternative (and equivalent) definition of $\mathcal{B}$-interpolation:

Definition. A sequence $\Lambda$ is free interpolation (denoted $\Lambda \in$ Int $_{\ell^{\infty}} \mathcal{B}$ ) if the trace $\mathcal{R}_{\Lambda}(\mathcal{B})$ is ideal, that is, if $\ell^{\infty} \cdot \mathcal{R}_{\Lambda}(\mathcal{B}) \subset \mathcal{R}_{\Lambda}(\mathcal{B})$.

Thus, $\Lambda \in$ Int $_{\ell^{\infty}} \mathcal{B}$ if and only if whenever $f \in \mathcal{B}$ and $\left\{\alpha_{\lambda}\right\} \in \ell^{\infty}$ there exists $g \in \mathcal{B}$ such that $g(\lambda)=\alpha_{\lambda} f(\lambda), \lambda \in \Lambda$. Since $\mathcal{B}$ is an algebra, this happens if and only if for any sequence of values $\left\{\alpha_{\lambda}\right\}_{\lambda \in \Lambda} \in \ell^{\infty}$ there exists $f \in \mathcal{B}$ with $f(\lambda)=\alpha_{\lambda}, \lambda \in \Lambda$.

There exists a general theory (and in particular an analytic description of interpolating sequences) for the algebras

$$
A_{p}=\left\{f \in \operatorname{Hol}(\mathbb{C}): \exists C>0: \sup _{z \in \mathbb{C}}|f(z)| e^{-C p(z)}<\infty\right\},
$$


where $p: \mathbb{C} \longrightarrow \mathbb{R}_{+}$is a subharmonic weight such that:

(a) $\log \left(1+|z|^{2}\right)=O(p(z))$,

(b) there are constants $C, D>0$ such that whenever $|\zeta-z| \leq 1$ then $p(\zeta) \leq C p(z)+D$

For this we refer to [BrGa95, Chapter 2].

Notice that $\mathcal{B}$ does not fall into this general theory, since $p(z)=|\operatorname{Im} z|$ does not satisfy (a) (in particular, $\mathcal{B}$ does not contain polynomials). However, the techniques used in the description of $A_{p}$-interpolation mentioned above (see [BrLi95, Corollary 3.5]) can be adapted to obtain an analogous result for $\mathcal{B}$.

Theorem A. The following statements are equivalent:

(a) $\Lambda \in$ Int $\mathcal{B}$

(b) $\Lambda \in \operatorname{Int}_{\ell \infty} \mathcal{B}$

(c) There exist $A, B, C>0$ and peak-functions $f_{\lambda} \in \mathcal{B}$ such that $f_{\lambda}\left(\lambda^{\prime}\right)=\delta_{\lambda, \lambda^{\prime}}$ and

$$
\sup _{z \in \mathbb{C}}\left|f_{\lambda}(z)\right| e^{-C|\operatorname{Im} z|} \leq A e^{B|\operatorname{Im} \lambda|} .
$$

(d) There exist $F \in \mathcal{B}$ and $\varepsilon, C>0$ such that $F(\lambda)=0$ for all $\lambda \in \Lambda$ and

$$
\left|F^{\prime}(\lambda)\right| \geq \varepsilon e^{-C|\operatorname{Im} \lambda|} .
$$

As soon as the analytic conditions of Theorem A hold we can proceed as in [MOO06] and obtain geometric conditions. That paper deals with $A_{p}$-interpolation for weights of the form

$$
p(z)=|\operatorname{Im} z|+\omega(|z|),
$$

being $\omega(t)$ a subadditive increasing continuous function, normalized with $\omega(0)=0$ such that $\log (1+t) \lesssim \omega(t)$ and $\int_{0}^{\infty} \frac{\omega(t)}{1+t^{2}} d t<\infty$. Taking $p(z)=|\operatorname{Im} z|$ (i.e. $\omega \equiv 0$ ) and using Theorem A we can mimic the proof of [MOO06, Theorem 1] and obtain a geometric characterisation.

In order to state this result consider the counting function $n_{\Lambda}(z, r)$ defined previously and the integrated version

$$
N_{\Lambda}(z, r)=\int_{0}^{r} \frac{n_{\Lambda}(z, t)-n_{\Lambda}(z, 0)}{t} d t+n_{\Lambda}(z, 0) \log r .
$$

Hereinafter we assume that $\Lambda \cap \mathbb{R}=\emptyset$; otherwise replace $\mathbb{R}$ by any the horizontal line that does not contain any of the points in $\Lambda$.

Theorem B. The sequence $\Lambda$ is $\mathcal{B}$-interpolation if and only if:

(i) There is $C>0$ such that

$$
N_{\Lambda}(\lambda,|\operatorname{Im} \lambda|) \leq C|\operatorname{Im} \lambda| \quad \forall \lambda \in \Lambda .
$$


(ii) The following Carleson-type condition holds

$$
\sup _{x \in \mathbb{R}} \sum_{\lambda \in \Lambda} \frac{|\operatorname{Im} \lambda|}{|x-\lambda|^{2}}<\infty .
$$

Since the Poisson kernel at $\lambda$ in the corresponding half-plane (upper halfplane if $\operatorname{Im} \lambda>0$ and lower half-plane when $\operatorname{Im} \lambda<0)$ is $P(\lambda, x)=$ $\frac{|\operatorname{Im} \lambda|}{|x-\lambda|^{2}}$, a restatement of condition (ii) is that the measure $\sum_{\lambda \in \Lambda} \delta_{\lambda}$ has bounded Poisson balayage.

The paper is structured as follows. In Section 2 we prove Theorem A and describe other analytic properties of $\mathcal{B}$-interpolation sequences which are relevant in the proof of Theorem B. For the sake of completeness, we briefly recall the proof of Theorem B in Section 3 .

A final remark about notation. $C$ will always denote a positive constant and its actual value may change from one occurrence to the next. $A=O(B)$ and $A \lesssim B$ mean that $A \leq c B$ for some $c>0$, and $A \simeq B$ is $A \lesssim B \lesssim A$.

\section{PRoOF OF THE ANALYTIC CONDITIONS}

Before the proof of Theorem A we need to spell out some of its consequences.

Lemma 1. Let $\Lambda$ be a discrete sequence in $\mathbb{C}$ for which (c) in Theorem $A$ holds. Then

(i) There exist $\alpha, \varepsilon>0$ such that the discs $D_{\lambda}:=D\left(\lambda, \delta_{\lambda}\right)$, with $\delta_{\lambda}=$ $\varepsilon e^{-\alpha|\operatorname{Im} \lambda|}$, are pairwise disjoint.

(ii) There exist $C>0$ such that for all $\beta>2$

$$
\sup _{z \in \mathbb{C}} \sum_{\lambda \in \Lambda} \frac{e^{-C|\operatorname{Im} \lambda|}}{1+|z-\lambda|^{\beta}}<\infty .
$$

When $\Lambda$ satisfies (i) we say that $\Lambda$ is weakly separated.

Proof. (i) Fix $\lambda \in \Lambda$ and consider any $\lambda^{\prime}$ such that $\left|\lambda-\lambda^{\prime}\right| \leq 1$. Then

$$
1=\left|f_{\lambda}(\lambda)-f_{\lambda}\left(\lambda^{\prime}\right)\right| \leq \sup _{\zeta \in D(\lambda, 1)}\left|f_{\lambda}^{\prime}(\zeta)\right|\left|\lambda-\lambda^{\prime}\right| .
$$

By the Cauchy estimates

$\left|f_{\lambda}^{\prime}(\zeta)\right| \leq \sup _{\eta:|\eta-\zeta|=1}\left|f_{\lambda}(\eta)\right| \leq \sup _{z \in D(\lambda, 2)}\left|f_{\lambda}(z)\right| \leq \sup _{z \in D(\lambda, 2)} A e^{-B|\operatorname{Im} \lambda|} e^{-C \alpha|\operatorname{Im} z|} \lesssim e^{-(B+C)|\operatorname{Im} \lambda|}$,

which yields the desired estimate.

(ii) This is an immediate consequence of (i): if $C \geq 2 \alpha$ we have

$$
\sum_{\lambda \in \Lambda} \frac{e^{-C|\operatorname{Im} \lambda|}}{1+|z-\lambda|^{\beta}} \lesssim \sum_{\lambda \in \Lambda} \int_{D_{\lambda}} \frac{e^{-(C-2 \alpha)|\operatorname{Im} \zeta|}}{1+|z-\zeta|^{\beta}} d m(\zeta) \lesssim \int_{\mathbb{C}} \frac{d m(\zeta)}{1+|z-\zeta|^{\beta}}
$$


Proof of Theorem A. $(a) \Rightarrow(b)$. Obvious.

$(b) \Rightarrow(c)$. Here we use the scheme of [BrGa95, Lemma 2.2.6.]. Consider the complete metric space $S=\left\{v=\left\{v_{\lambda}\right\}_{\lambda}:\|v\|_{\infty} \leq 1\right\}$, and for $n \in \mathbb{N}$ let:

$S_{n}=\left\{\left\{v_{\lambda}\right\}_{\lambda} \in S: \exists f \in \mathcal{B}: f(\lambda)=v_{\lambda}, \lambda \in \Lambda\right.$, and $\left.\sup _{z \in \mathbb{C}}|f(z)| e^{-n|\operatorname{Im} z|} \leq n\right\}$.

By hypotheses $S=\cup_{n} S_{n}$ and by Baire's Cathegory Theorem there exists $n \in \mathbb{N}$ such that $\stackrel{\circ}{S}_{n} \neq \emptyset$. Since $0 \in S_{n}$ for all $n$, there exists $\varepsilon \in(0,1)$ such that

$$
\|v\|_{\infty} \leq \varepsilon \Longrightarrow v \in S_{n}
$$

Let $v^{\lambda}=\left\{\varepsilon \delta_{\lambda, \lambda^{\prime}}\right\}_{\lambda^{\prime}}$. There exists then $g_{\lambda} \in \mathcal{B}$ with $g_{\lambda}\left(\lambda^{\prime}\right)=\varepsilon \delta_{\lambda, \lambda^{\prime}}$ and $\sup _{z}\left|g_{\lambda}(z)\right| e^{-n|\operatorname{Im} z|} \leq n$. Taking $f_{\lambda}=g_{\lambda} / \varepsilon$ we get the desired properties.

$(c) \Rightarrow(d)$. Define $F(z)=\sum_{\lambda \in \Lambda} w_{\lambda}(z) g_{\lambda}(z)$, where

$$
w_{\lambda}(z)=\left(\frac{\sin (z-\lambda)}{z-\lambda}\right)^{3} e^{i \sigma_{\lambda} M(z-\lambda)}, \quad g_{\lambda}(z)=f_{\lambda}^{2}(z) \sin (z-\lambda),
$$

$M>0$ will be chosen later on and

$$
\sigma_{\lambda}=\left\{\begin{array}{cl}
1 & \text { if } \operatorname{Im} \lambda<0 \\
-1 & \text { if } \operatorname{Im} \lambda>0
\end{array}\right.
$$

Notice that $w_{\lambda}$ is a holomorphic weight in $\mathcal{B}$ with $w_{\lambda}(\lambda)=1$, while $g_{\lambda}$ is a function in $\mathcal{B}$ vanishing at order 1 on $\lambda$ and at least at order 2 on all $\lambda^{\prime} \neq \lambda$.

Let's see first that $F \in \mathcal{B}$. A direct estimate shows that

$$
\begin{aligned}
\left|w_{\lambda}(z)\right| & \lesssim \frac{e^{(M+3)|\operatorname{Im} z|} e^{-(M-3)|\operatorname{Im} \lambda|}}{1+|z-\lambda|^{3}}, \\
\left|g_{\lambda}(z)\right| & \lesssim e^{(2 C+1)|\operatorname{Im} z|} e^{(2 B+1)|\operatorname{Im} \lambda|},
\end{aligned}
$$

and therefore

$$
|F(z)| \lesssim e^{(M+2 C+4)|\operatorname{Im} z|} \sum_{\lambda \in \Lambda} \frac{e^{-(M-2 B-4)|\operatorname{Im} \lambda|}}{1+|z-\lambda|^{3}} .
$$

Taking $M$ big enough and applying Lemma1(ii) we see that $F \in \mathcal{B}$.

It is clear that $F(\lambda)=0$ for all $\lambda \in \Lambda$. On the other hand

$$
F^{\prime}(\lambda)=\sum_{\lambda^{\prime} \in \Lambda} w_{\lambda^{\prime}}(\lambda) g_{\lambda^{\prime}}^{\prime}(\lambda)=w_{\lambda}(\lambda) g_{\lambda}^{\prime}(\lambda)=1 .
$$

$(d) \Rightarrow(a)$. Let $\left\{v_{\lambda}\right\}_{\lambda \in \Lambda}$ satisfy (11) and define

$$
f(z)=\sum_{\lambda \in \Lambda} v_{\lambda} w_{\lambda}(z) \frac{F(z)}{F^{\prime}(\lambda)(z-\lambda)},
$$

where $w_{\lambda}$ is defined as before. 
It is clear that $F(\lambda)=v_{\lambda}$ for all $\lambda \in \Lambda$, and a similar computation to the one in the previous implication shows that $f \in \mathcal{B}$, since $\mid F(z) /\left(F^{\prime}(\lambda)(z-\right.$ $\lambda)) \mid \lesssim e^{C(|\operatorname{Im} z|+|\operatorname{Im} \lambda|)}$.

We finish this section by showing that a weakly separated union of two $\mathcal{B}$-interpolation sequences is still $\mathcal{B}$-interpolation.

Theorem 2. Let $\Lambda_{1}, \Lambda_{2} \in \operatorname{Int} \mathcal{B}$ be such that $\Lambda_{1} \cup \Lambda_{2}$ is weakly separated. Then $\Lambda_{1} \cup \Lambda_{2} \in \operatorname{Int} \mathcal{B}$.

For the proof of this result we will need the following lemma.

Lemma 3. Let $\Lambda \in \operatorname{Int} \mathcal{B}$ and let $z_{0} \notin \Lambda$. There exist $A, B, C>0$ independent of $z_{0}$ and $f \in \mathcal{B}$ such that

(i) $f\left(z_{0}\right)=1$ and $f(\lambda)=0$ for all $\lambda \in \Lambda$.

(ii) $\sup _{z \in \mathbb{C}}|f(z)| e^{-C|\operatorname{Im} z|} \leq B e^{-C\left|\operatorname{Im} z_{0}\right|} / d\left(z_{0}, \Lambda\right)$.

Proof. Assume first that $z_{0}=0$. Since $\Lambda$ is weakly separated there exists $\delta>0$ be such that $\left|\lambda-\lambda^{\prime}\right| \geq 10 \delta$ for all $\lambda, \lambda^{\prime} \in \Lambda \cap\{|\operatorname{Im} z|<1\}$.

Lemma 4. There exists $F \in \mathcal{B}$ with $F(0)=0$ and such that

$$
\begin{array}{ll}
|F(\lambda)| \geq \varepsilon & \text { if }|\lambda| \geq \delta \\
|F(\lambda)| \geq \varepsilon|\lambda| & \text { if }|\lambda|<\delta .
\end{array}
$$

We deferr the proof of this technichal result to the end of the section.

Consider the values $v_{\lambda}=1 / F(\lambda)$. Since $\sup _{\lambda \in \Lambda}\left|v_{\lambda}\right| \leq 1 / \min (\varepsilon, \varepsilon d(0, \Lambda))$, there exist $A, B>0$ and $G \in \mathcal{B}$ such that $G(\lambda)=v_{\lambda}=1 / F(\lambda)$ and $\sup _{z \in \mathbb{C}}|G(z)| e^{-A|\operatorname{Im} z|} \leq B /(\varepsilon d(0, \Lambda))$.

The function $f(z)=1-F(z) G(z)$ satisfies then the required properties.

For $z_{0} \notin \Lambda$ arbitrary apply the previous case to the sequence $\Lambda-z_{0}$. Notice that $\Lambda-z_{0} \in \operatorname{Int} \mathcal{B}$, since there exist the peak-functions required by Theorem $\mathrm{A}(\mathrm{c})$ : just take $f_{\lambda-z_{0}}(z)=f_{\lambda}\left(z+z_{0}\right)$, where $f_{\lambda}$ are the peakfunctions associated to $\Lambda$.

Proof of Theorem 2 Let us show that there exist peak-functions as in Theorem $\mathrm{A}(\mathrm{c})$.

Fix $\lambda_{1} \in \Lambda_{1}$. Applying the previous Lemma to $\Lambda_{2}$ and $z_{0}=\lambda_{1}$ we see that there exist $A, B>0$ and $g_{\lambda_{1}} \in \mathcal{B}$ such that

$$
\begin{aligned}
& g_{\lambda_{1}}\left(\lambda_{1}\right)=1, \quad \text { and } \quad g_{\lambda_{1}}\left(\lambda_{2}\right)=0 \forall \lambda_{2} \in \Lambda_{2} \\
& \sup _{z \in \mathbb{C}}\left|g_{\lambda_{1}}(z)\right| e^{-A|\operatorname{Im} z|} \leq B \frac{e^{C\left|\operatorname{Im} \lambda_{1}\right|}}{d\left(\lambda_{1}, \Lambda_{2}\right)} \leq B e^{C^{\prime}\left|\operatorname{Im} \lambda_{1}\right|} .
\end{aligned}
$$

Since $\Lambda_{1} \in \operatorname{Int} \mathcal{B}$ there exist a functions $f_{\lambda_{1}} \in \mathcal{B}$, as given by Theorem A(c). Then $h_{\lambda_{1}}=f_{\lambda_{1}} g_{\lambda_{1}}$ is a peak-function for $\Lambda_{1} \cup \Lambda_{2}$. 
Proof of Lemma 4 Consider

$$
\sin (\pi z)=\pi z \prod_{k \in \mathbb{Z} \backslash\{0\}}\left(1-\frac{z}{k}\right) .
$$

If $|\lambda-k| \geq \delta$ for all $\lambda \in \Lambda$ and $k \in \mathbb{Z}$, we can just take $F(z)=\sin (\pi z)$.

For each $k \in \mathbb{N}$ such that there this $\lambda \in \Lambda$ with $|\lambda-k|<\delta$ or $|\lambda+k|<\delta$ we replace in $\sin (\pi z)$ the factors $1-z / k, 1+z / k$ by small perturbations $1-z / p_{k}, 1-z / p_{-k}$, where $p_{k}=k+i \epsilon_{k}$ and $\epsilon_{k}>0$ is chosen so that

(a) $\left|\epsilon_{k}\right| \leq 5 \delta$

(b) $\epsilon_{-k}=\epsilon_{k}$

(c) $\left|p_{k}-\lambda\right| \geq \delta$ and $\left|p_{-k}-\lambda\right| \geq \delta$ for all $\lambda \in \Lambda$.

Denoting by $\mathcal{I}$ the indices of $\mathbb{Z} \backslash\{0\}$ affected by this modification, we define

$$
F(z)=\sin (\pi z) \prod_{k \in \mathcal{I}} \frac{1-z / p_{k}}{1-z / k} .
$$

Notice that the zeros of this new sine-type function are at a distance of $\Lambda$ no smaller that $\delta$.

In order to see that $F \in \mathcal{B}$ is is enough to show that the latter product is bounded above and below away from the points $k$ and $p_{k}$. To do so we group the factors corresponding to $k$ and $-k$. Since $p_{-k}=-\overline{p_{k}}$ we have

$$
\begin{aligned}
\prod_{k \in \mathcal{I}} \frac{1-z / p_{k}}{1-z / k} & =\prod_{k \in \mathcal{I}} \frac{z-p_{k}}{z-k} \frac{k}{p_{k}}=\prod_{k \in \mathcal{I} \cap \mathbb{N}} \frac{\left(z-p_{k}\right)\left(z-p_{-k}\right)}{(z-k)(z+k)} \frac{-k^{2}}{p_{k} p_{-k}} \\
& =\prod_{k \in \mathcal{I} \cap \mathbb{N}}\left(1-\frac{\epsilon_{k}\left(\epsilon_{k}+2 i z\right)}{z^{2}-k^{2}}\right) \frac{1}{1+\epsilon_{k}^{2} / k^{2}},
\end{aligned}
$$

from which the required properties follow easily.

\section{Proof of THE GeOMETRIC CONDITIONS}

In this section we briefly recall the proof of Theorem B.

3.1. Necessity. The necessity of (i) is a standard (and straightforward) application of Jensen's formula to the functions $f_{\lambda}$ provided by Theorem $\mathrm{A}(\mathrm{c})$ on the disks $D(\lambda,|\operatorname{Im} \lambda|)$.

To see that (ii) is also necessary consider the functions $g_{\lambda}(z)=f_{\lambda}(z) e^{i C z}$, which according to Theorem $\mathrm{A}(\mathrm{c})$ are bounded in the upper half-plane $\mathbb{H}$. Applying Jensen's formula to $g_{\lambda}$ in $\mathbb{H}$ we have

$$
\sum_{\lambda^{\prime} \neq \lambda: \operatorname{Im} \lambda^{\prime}>0} \log \left|\frac{\lambda-\lambda^{\prime}}{\lambda-\bar{\lambda}^{\prime}}\right|^{-1} \lesssim \operatorname{Im} \lambda \quad \text { for all } \quad \lambda \in \Lambda \cap \mathbb{H}
$$


The estimate $1-t \leq \log t^{-1}$ for $t \in(0,1)$ yields then

$$
\sup _{\lambda \in \Lambda \cap \mathbb{H}} \sum_{\lambda^{\prime} \neq \lambda: \operatorname{Im}} \frac{\operatorname{Im} \lambda^{\prime}>0}{\left|\lambda-\bar{\lambda}^{\prime}\right|^{2}}<+\infty .
$$

Given $x \in \mathbb{R}$, consider the $\lambda \in \Lambda \cap \mathbb{H}$ closest to $x$. Then $\frac{\operatorname{Im} \lambda^{\prime}}{\left|x-\lambda^{\prime}\right|^{2}} \leq$ $2 \frac{\operatorname{Im} \lambda^{\prime}}{\left|\lambda-\lambda^{\prime}\right|^{2}}$. This and the corresponding computation for the lower half-plane imply (ii).

3.2. Sufficency. According to Theorem 2 it will be enough to show that $\Lambda_{+}=\Lambda \cap\{\operatorname{Im} z>0\}$ and $\Lambda_{-}=\Lambda \cap\{\operatorname{Im} z<0\}$ are $\mathcal{B}$-interpolation.

By Theorem $\mathrm{A}(\mathrm{d})$, in order to prove that $\Lambda_{+}$is $\mathcal{B}$-interpolation it is enough to construct a function $F \in \mathcal{B}$ such that $\Lambda_{+} \subset \mathcal{Z}(F)$ and

$$
\left|F^{\prime}(\lambda)\right| \geq \varepsilon e^{-K \operatorname{Im} \lambda} \quad \lambda \in \Lambda_{+}
$$

for some constants $\varepsilon, k>0$.

Start with any entire function $G$ with zero ser $\mathcal{Z}(G)=\Lambda_{+}$. Condition (ii) implies that $\Lambda_{+}$is a Blaschke sequence in $\mathbb{H}$, i.e. the Blaschke product

$$
B(z)=\prod_{\lambda \in \Lambda_{+}} \frac{z-\lambda}{z-\bar{\lambda}}, \quad z \in \mathbb{H}
$$

converges. Define

$$
\Psi(z)=N|\operatorname{Im} z|-\phi(z)
$$

where

$$
\phi(z)= \begin{cases}\log \left|\frac{G(z)}{B(z)}\right| & \operatorname{Im} z>0 \\ \log |G(z)| & \operatorname{Im} z \leq 0 .\end{cases}
$$

A computation shows that $\phi$ is harmonic outside the real axis, subharmonic on $\mathbb{C}$ and $\Delta \phi(x)=\sum_{\lambda \in \Lambda_{+}} \frac{\operatorname{Im} \lambda}{|x-\lambda|^{2}} d x$ (see [MO006, Lemma 10]). Thus, by condition (ii), $\Delta \Psi \simeq d x$ when $N \in \mathbb{N}$ is big enough. In this situation, according to [OS99, Lemma 3], there exists a multiplier associated to $\Psi$, i.e., an entire function $h$ such that:

(a) $\mathcal{Z}(h)$ is a separated sequence contained in $\mathbb{R}$ and separated from $\Lambda$ (i.e. $\left.\inf _{z \in \mathcal{Z}(h)} d(z, \Lambda)>0\right)$.

(b) Given any $\varepsilon>0,|h(z)| \simeq \exp (\Psi(z))$ for all points $z$ such that $d(z, \mathcal{Z}(h))>\varepsilon$.

Define now $F=h G$. It is clear that $F \in \mathcal{B}$ :

$$
|F(z)| \lesssim e^{\Psi(z)+\log |G(z)|} \leq e^{\Psi(z)+\phi(z)} \leq e^{N p(z)} \quad z \in \mathbb{C} .
$$

It is also clear that $\Lambda_{+} \subset \mathcal{Z}(F)$, since $\Lambda_{+} \subset \mathcal{Z}(G)$. 
In order to prove that there exist $\varepsilon, C>0$ such that

$$
\left|F^{\prime}(\lambda)\right| \geq \varepsilon e^{-C \operatorname{Im} \lambda}
$$

consider the disjoint disks $D_{\lambda}=D\left(\lambda, \delta_{\lambda}\right), \delta_{\lambda}=\delta e^{-C \frac{p(\lambda)}{m_{\lambda}}}$ provided by Lemma 1 (i). Since $\Lambda_{+}$is far from $\mathcal{Z}(h)$, the estimate

$$
|G(z)|=|h(z)| e^{\phi(z)}|B(z)| \simeq e^{N|\operatorname{Im} z|}|B(z)| \quad z \in \partial D_{\lambda}
$$

holds.

From here we finish as in [MOO06]: since the hypotheses imply that there exists $C>0$ such that $|B(z)| \geq \epsilon e^{-C|\operatorname{Im} z|}, z \in \partial D_{\lambda}$, we have $|F(z)| \gtrsim e^{-C p(z)}$ for all $z \in \partial D_{\lambda}$. The function $g(z)=F(z) /(z-\lambda)$ is then holomorphic, non-vanishing in $D_{\lambda}$, and with $|g(z)| \gtrsim e^{-c \operatorname{Im} \lambda}$ for $z \in \partial D_{\lambda}$. By the minimum principle

$$
\left|F^{\prime}(\lambda)\right|=|g(0)| \gtrsim e^{-c \operatorname{Im} \lambda}
$$

as desired.

\section{REFERENCES}

[BrGa95] Berenstein, C.A. - Gay, R. Complex Analysis and Special Topics in Harmonic Analysis, Springer Verlag, 1995. MR 96j:30001.

[BrLi95] C.A. Berenstein and B.Q. Li, Interpolating varieties for spaces of meromorphic functions, J. Geom. Anal. 5 (1995), 1-48. MR 96b:30064.

[Fa07] Favorov, S. Ju. Zero sets of entire functions of exponential type with some conditions on the real axis, Preprint, 2007. arXiv:math.CV/0701808v1.

[MOO06] X. Massaneda, J. Ortega-Cerdà and M. Ounaïes. A geometric characterization of interpolation in $\hat{\mathcal{E}}^{\prime}(\mathbb{R})$, Trans. Amer. Math. Soc. 358 (2006), 3459-3472. MR 2006m:30063.

[OS99] J. Ortega-Cerdà and K. Seip. Multipliers for entire functions and an interpolation problem of Beurling, J. Funct. Anal. 162 (1999), 400-415. MR 2000c:30071.

Departament de Matemàtica Aplicada i AnÀlisi, Universitat de BarceLONA, GRAN Via 585, 08071-BARCELONA, SPAIN

E-mail address: xavier.massaneda@ub.edu

Departament de Matemàtica Aplicada i ANÀlisi, Universitat de BarceLONA, GRAN VIA 585, 08071-BARCELONA, SPAIN

E-mail address: jortega@ub.edu 